\title{
HIGH-ORDER MULTISCALE FINITE ELEMENT METHOD FOR ELLIPTIC PROBLEMS*
}

\author{
JAN S. HESTHAVEN ${ }^{\dagger}$, SHUN ZHANG ${ }^{\ddagger}$, AND XUEYU ZHU§
}

\begin{abstract}
In this paper, a new high-order multiscale finite element method (MsFEM) is developed for elliptic problems with highly oscillating coefficients. The method is inspired by the MsFEM developed in [G. Allaire and R. Brizzi, Multiscale Model. Simul., 4 (2005), pp. 790-812], but a more explicit multiscale finite element space is constructed. The approximation space is nonconforming when an oversampling technique is used. We use a Petrov-Galerkin formulation suggested in [T. Y. Hou, X.-H. Wu, and Y. Zhang, Commun. Math. Sci., 2 (2004), pp. 185-205] to simplify the implementation and to improve the accuracy. The method is natural for high-order finite element methods used with the advantage of solving the coarse grained problem. We prove optimal error estimates in the case of periodically oscillating coefficients and support the findings by various numerical experiments.
\end{abstract}

Key words. scalar elliptic partial differential equation, multiscale finite element method, highorder accuracy, oversampling, Petrov-Galerkin

AMS subject classifications. 65N12, 65N30, 65M60

DOI. $10.1137 / 120898024$

1. Introduction. The development of numerical methods for problems with highly oscillating coefficients is an increasingly active field of research. To overcome the computational cost of resolving the fine scale, multiscale finite element methods (MsFEMs) have been developed in [12, 13, 11, 8, 14, 10]. Accuracy is achieved by solving a fine scale problem locally. These solutions are used to build the multiscale finite element basis to capture the small scale information of the leading-order differential operator. There are several alternatives to this approach for multiscale methods, for example, the multiscale variational method [15] and the heterogeneous multiscale method (HMM) [1], and additional methods are discussed in [4, 19, 7]. In this work, however, we focus on techniques based on multiscale finite element formulations.

Originally, MsFEMs were proposed for linear finite elements; see [12, 13]. For many applications, e.g., elliptic problems with singular forcing or nonconvex domains, and wave equations, high-order finite elements are known to be advantageous in terms of accuracy and efficiency, in particular for large problems. Allaire and Brizzi [3] generalized the original approach to enable the use of high-order (order higher than one) elements by local harmonic coordinates. This method uses a composite rule to change the local coordinates. Inspired by that work, we propose in this work a new high-order accurate MsFEM. However, in contrast to the previous work, we do not use a composite rule but approach the development in a more explicit way. Additionally,

${ }^{*}$ Received by the editors November 7, 2012; accepted for publication (in revised form) February 10, 2014; published electronically May 22, 2014. This work was partially supported by OSD/AFOSR FA9550-09-1-0613.

http://www.siam.org/journals/mms/12-2/89802.html

${ }^{\dagger}$ Chair of Computational Mathematics and Simulation Science (MCSS), Mathematics Institute of Computational Science and Engineering (MATHICSE), Ecole Polytechnique Fédérale de Lausanne (EPFL), CH-1015 Lausanne, Switzerland, and Division of Applied Mathematics, Brown University, Providence, RI 02912 (Jan.Hesthaven@epfl.ch).

${ }^{\ddagger}$ Department of Mathematics, City University of Hong Kong, Kowloon Tong, Hong Kong, China (shun.zhang@cityu.edu.hk).

$\S$ Scientific Computing and Imaging Institute, University of Utah, Salt Lake City, UT 84112 (xzhu@sci.utah.edu). 
we also solve local oscillating functions by high-order finite elements, as is necessary in many cases to preserve the accuracy. This overall approach proves itself to be cheaper to implement. To further improve the accuracy and simplify the implementation, an oversampling technique and Petrov-Galerkin formulations following [11, 14] are used. The bases are nonconforming when the oversampling technique is used. Similar to the method proposed in [3], the analysis is restricted to the periodic case, but the method is derived and applied to the general nonperiodic cases. Note that the analysis in [14] assumes that the local problem is solved exactly in $H^{1}$. In this paper, we improve these results to the more practical situation in which we assume that the local problem is solved by some high-order finite elements as in [3]. New discussion of the harmonic coordinate in the multiscale method can be found in [19], where the globally defined harmonic coordinate is solved. Other high-order methods for generalized finite element methods are discussed in [17].

The rest of this paper is organized as follows. The formulations of the model elliptic problem and the motivation for the new MsFEM are discussed in section 2. The new high-order MsFEM is introduced in section 3. In section 4, convergence results are proved for the periodic case. Implementations and numerical experiments are discussed in section 5, and section 6 contains a few final remarks and the outlook for continued work.

2. Model problem and motivations. Let $\Omega$ be a bounded polygonal domain in $\mathbb{R}^{n}$, and consider the elliptic model problem:

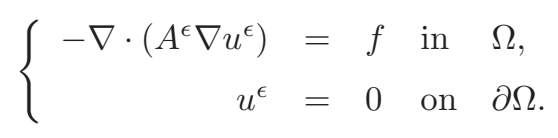

The matrix $A^{\epsilon} \in L^{\infty}\left(\Omega, \mathcal{M}_{\alpha_{A}, \gamma_{A}}\right)$, where $\mathcal{M}_{\alpha_{A}, \gamma_{A}}$ is the set of uniformly positive definite matrices with uniformly positive definite inverse; that is, for any $\xi \in \mathbb{R}^{d}$, $\|\xi\|_{\ell^{2}}=1,0<\alpha_{A} \leq \xi^{T} A^{e p s} \xi \leq \gamma_{A}^{-1}$. In the periodic case we have

$$
A^{\epsilon}(x)=A\left(\frac{x}{\epsilon}\right)
$$

where $y \mapsto A(y)$ is a $Y$-periodic function with $Y=(0,1)^{n}$.

Let $\chi_{i}, i=1, \ldots, n$, be the solution to the cell problem

$$
\begin{cases}-\operatorname{div}_{y}\left(A(y) \nabla_{y} \chi_{i}\right)=\operatorname{div}_{y}\left(A(y) e_{i}\right) & \text { in } Y, \\ y \mapsto x_{i}(y) & Y \text {-periodic. }\end{cases}
$$

The notation $\langle\cdot\rangle_{Y}$ is used to denote the mean of a function in domain $Y$ :

$$
\langle f\rangle_{Y}=\frac{1}{Y} \int_{Y} f(x) d x
$$

From classical homogenization theory (see, e.g., $[5,9,2,18,20]$ ) we have the following approximation results:

$$
u^{\epsilon}(x) \approx u^{*}(x)+\epsilon \sum_{i=1}^{n} \chi_{i}\left(\frac{x}{\epsilon}\right) \frac{\partial u^{*}}{\partial x_{i}}(x)
$$

and

$$
\nabla u^{\epsilon}(x) \approx \nabla u^{*}(x)+\epsilon \sum_{i=1}^{n}\left(\nabla_{y} \chi_{i}\right)\left(\frac{x}{\epsilon}\right) \frac{\partial u^{*}}{\partial x_{i}}(x) .
$$


Here $u^{*}$ is the solution of the homogenization problem

$$
\left\{\begin{aligned}
-\nabla \cdot\left(A^{*} \nabla u^{*}\right) & =f \text { in } \Omega, \\
u^{*} & =0 \text { on } \partial \Omega,
\end{aligned}\right.
$$

where $A^{*}$ is a constant homogenized matrix given by the explicit formula

$$
A^{*} e_{i}=\int_{Y} A(y)\left(e_{i}+\nabla_{y} \chi_{i}\right) d y \quad \text { with } e_{i} \text { being the } i \text { th canonical basis of } \mathbb{R}^{n} .
$$

Formula (2.3) looks like a first-order Taylor expansion of the map

$$
u^{\epsilon}(x) \approx u^{*}\left(x+\epsilon \chi\left(\frac{x}{\epsilon}\right)\right) .
$$

Based on this observation, [3] introduces a multiscale finite element basis using the composition rule,

$$
\Phi^{\epsilon, h}(x)=\Phi^{h}\left(\hat{w}^{\epsilon, h}\left(\frac{x}{\epsilon}\right)\right),
$$

where $\Phi^{h}$ is a standard finite element basis function on a coarse mesh $h>\epsilon$ and $\hat{w}^{\epsilon, h}$ is a numerical solution of a local multiscale problem on an element $K$ or an oversampled domain $S$ containing $K$ with finite element spaces defined on local meshes on $K$ or $S$. For the periodic case, $\hat{w}^{\epsilon}$ is a numerical approximation of $\epsilon \chi\left(\frac{x}{\epsilon}\right)-x$.

Taking a second look at (2.3), ignoring the apparent connection to a Taylor expansion or composite rule, we propose the following multiscale finite element basis functions:

$$
\Phi^{\epsilon, h}(x)=\Phi^{h}+\left(\hat{w}^{\epsilon, h}\left(\frac{x}{\epsilon}\right)-x\right) \cdot \nabla \Phi^{h} .
$$

This new multiscale finite element basis will clearly lead to a nonconforming element method when oversampling is used. However, as we shall discuss, this will not be an issue for either the analysis or the implementation. When both $\Phi^{h}$ and $\hat{w}^{\epsilon, h}$ are of high order in order to maintain the accuracy, the new formulation is cheaper to implement in the sense that its polynomial degree is lower.

3. MsFEM. In this section, we introduce and derive the details of the MsFEM for the general nonperiodic case.

For simplicity of the presentation, we consider only triangular elements. Let $\mathcal{T}_{h}=\{K\}$ be a finite element partition of the domain $\Omega$. Assume that the triangulation $\mathcal{T}_{h}$ is regular and quasi-uniform with size $h$. Let $P_{k}(K)$ be the space of polynomials of degree $k$ on element $K$.

Without confusion, we will use $v=\left(v_{1}, \ldots, v_{n}\right)$ to denote an $n$-dimensional vector. Then $\nabla v$ is a matrix, and we denote it by $[\nabla v]$ to emphasize it is a matrix.

3.1. Local element problems. Let us introduce the following local problem as that in [3]:

$$
\left\{\begin{aligned}
-\nabla \cdot\left(A^{\epsilon} \nabla \hat{w}_{i}^{\epsilon, K}\right) & =-\nabla \cdot\left(A_{K}^{*} \nabla x_{i}\right) & & \text { in } \quad K, \\
\hat{w}_{i}^{\epsilon, K} & =x_{i} & & \text { on } \partial K .
\end{aligned}\right.
$$


If the homogenized matrix $A^{*}$ is a constant matrix, or $A^{*}$ is piecewise smooth, and the interface of discontinuity is aligned with the mesh, when the size of $K$ is small enough (when it is piecewise constant, $K$ need not be too small), we can take $A_{K}^{*}$ as a local approximation of $A^{*}$ to be a constant matrix in $K$. In this case, $\nabla \cdot\left(A_{K}^{*} \nabla x_{i}\right)=0$ in $K$.

Thus, we introduce the simplified local problem: For each $K \in \mathcal{T}_{h}$, define $\hat{w}_{i}^{\epsilon, K}$, $i=1, \ldots, n$, as the solution of

$$
\left\{\begin{aligned}
-\nabla \cdot\left(A^{\epsilon} \nabla \hat{w}_{i}^{\epsilon, K}\right) & =0 \quad \text { in } \quad K, \\
\hat{w}_{i}^{\epsilon, K} & =x_{i} \text { on } \partial K .
\end{aligned}\right.
$$

We will use (3.1) if a better nonconstant approximation of $A^{*}$ is known. The vector $\hat{w}^{\epsilon}$ is defined as $\hat{w}^{\epsilon}=\left(\hat{w}_{1}^{\epsilon}, \cdots, \hat{w}_{n}^{\epsilon}\right) \in H^{1}(\Omega)^{n}$, where $\hat{w}_{i}^{\epsilon} \in H^{1}(\Omega)$ with $\hat{w}_{i}^{\epsilon}=\hat{w}_{i}^{\epsilon, K}$ for each $K \in \mathcal{T}_{h}$.

For each $K \in \mathcal{T}$, a quasi-uniform fine mesh $\mathcal{T}_{h^{\prime}}(K)$ with element size $h^{\prime}$ is introduced. Define

$$
W_{h^{\prime}}(K)=\left\{w \in C^{0}(K):\left.w\right|_{T} \in P_{k^{\prime}}(T) \forall T \in \mathcal{T}_{h^{\prime}}(K)\right\},
$$

and let $w_{i}^{\epsilon, K}$ be the $P_{k^{\prime}}$ finite element approximation of $\hat{w}_{i}^{\epsilon, K}$ in (3.2) using mesh $\mathcal{T}_{h^{\prime}}^{K}$. Find $w_{i}^{\epsilon, K} \in W_{h^{\prime}}(K), w_{i}^{\epsilon, K}=x_{i}$ on $\partial K$, such that

$$
\left(A^{\epsilon} \nabla \hat{w}_{i}^{\epsilon, K}, \nabla v\right)=0, \quad v \in W_{h^{\prime}}(K) \cap H_{0}^{1}(K) .
$$

Define $w^{\epsilon, h}=\left(w_{1}^{\epsilon, h}, \ldots, w_{n}^{\epsilon, h}\right) \in H^{1}(\Omega)^{n}$, where $w_{i}^{\epsilon, h} \in H^{1}(\Omega)$ with $w_{i}^{\epsilon, h}=w_{i}^{\epsilon, K}$ for each $K \in \mathcal{T}_{h}$.

3.1.1. Oversampled local problem. In order to remove the resonance effect [10] associated with the lack of correct boundary conditions on the local problem, we shall use an oversampling method on a domain $S \supset K$. Define $\hat{w}_{i}^{\epsilon, S}, i=1, \ldots, n$, as the solution of

$$
\left\{\begin{aligned}
-\nabla \cdot\left(A^{\epsilon} \nabla \hat{w}_{i}^{\epsilon, S}\right) & =-\nabla \cdot\left(A_{S}^{*} \nabla x_{i}\right) & & \text { in } S, \\
\hat{w}_{i}^{\epsilon, S} & =x_{i} & & \text { on } \partial S .
\end{aligned}\right.
$$

When the size of $S$ is small enough and there is no discontinuity of $A^{*}$ inside $S$, we take $A_{S}^{*}$, a local approximation of $A^{*}$, to be a constant matrix in $S$. In this case, the right-hand side of (3.4) is 0:

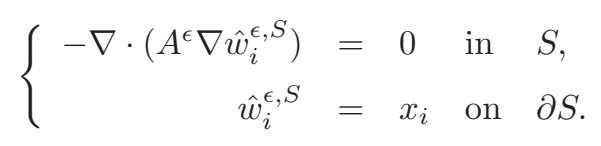

We define $\hat{w}_{i}^{\epsilon, K}=\left.\hat{w}_{i}^{\epsilon, S}\right|_{K}, i=1, \ldots, n$, and define $\hat{w}^{\epsilon}$ accordingly. In general, $\hat{w}^{\epsilon}$ obtained from the oversampling method is not in $H^{1}(\Omega)^{n}$.

For a polygonal domain $S \supset K$, define a quasi-uniform fine mesh $\mathcal{T}_{h^{\prime}}(S)$ with element size $h^{\prime}$. The edges of the mesh are aligned with the sides of $K$. Define

$$
W_{h^{\prime}}(S)=\left\{w \in C^{0}(S):\left.w\right|_{T} \in P_{k^{\prime}}(T) \forall T \in \mathcal{T}_{h^{\prime}}(S)\right\},
$$

and let $w_{i}^{\epsilon, S}$ be the $P_{k^{\prime}}$ finite element approximation of $\hat{w}_{i}^{\epsilon, S}$ of (3.5) using mesh $\mathcal{T}_{h^{\prime}}(S)$. Find $w_{i}^{\epsilon, S} \in W_{h^{\prime}}(S), w_{i}^{\epsilon, S}=x_{i}$ on $\partial S$, such that

$$
\left(A^{\epsilon} \nabla \hat{w}_{i}^{\epsilon, S}, \nabla v\right)=0 \quad \forall v \in W_{h^{\prime}}(S) \cap H_{0}^{1}(S) .
$$


With $w^{\epsilon, K}=\left.w^{\epsilon, S}\right|_{K}$, we can define $w^{\epsilon, h}$ accordingly. Like $\hat{w}^{\epsilon}$, in general, $w^{\epsilon, h}$ is not an $H^{1}$ vector function.

As generally practiced, in the analysis of this paper and in our numerical tests, we will use the zero right-hand side versions of the local problems. We also assume that the local problem is solved by a large enough oversampling domain $S$.

3.1.2. Homogenization results for the local problem. It is known [5] that the solution to (3.5) has the following structure:

$$
\hat{w}_{i}^{\epsilon, S}=\hat{w}_{i}^{0, S}+\epsilon \chi(x / \epsilon) \cdot \nabla \hat{w}_{i}^{0, S}+\epsilon \theta_{i}^{\epsilon, S},
$$

where $\hat{w}_{i}^{0, S} \in H^{2}(S)$ is the solution of the homogenized equation

$$
-\nabla \cdot\left(A^{*} \nabla \hat{w}_{i}^{0, S}\right)=0 \quad \text { in } S,
$$

with boundary condition $\hat{w}_{i}^{0, S}=x_{i}$ on $\partial S$. One easily shows that $\hat{w}_{i}^{0, S}=x_{i}$ on the whole element $S$. Thus

$$
\hat{w}_{i}^{\epsilon, S}=x_{i}+\epsilon \chi(x / \epsilon)_{i}+\epsilon \theta_{i}^{\epsilon, S} .
$$

For a nonoversampled method, $K=S$, while for the oversampling method, we assume that for $\theta_{i}^{\epsilon, K}=\left.\left(\theta_{i}^{\epsilon, S}\right)\right|_{K}$ the following holds.

Assumption 3.1 (see [14, Assumption 2.1]). The oversampling domain $S$ is chosen such that for any element $K$ in $S$,

$$
\left\|\nabla \theta_{i}^{\epsilon, K}\right\|_{L^{\infty}(K)} \leq C
$$

where $C$ is a constant independent of $\epsilon$ and $h$.

For the periodic problem, define

$$
\tilde{w}_{i}^{\epsilon}(x)=x_{i}+\epsilon \chi_{i}\left(\frac{x}{\epsilon}\right), \quad i=1, \ldots, n,
$$

and denote $\tilde{w}=\left(\tilde{w}_{1}, \ldots, \tilde{w}_{n}\right)$.

Let $\hat{w}^{\epsilon, h}$ and $w^{\epsilon, h}$ be the solutions of (3.2) and (3.6), respectively. We have the following estimates (see Theorem 4.1 of [3]):

$$
\left\|\nabla\left(\tilde{w}^{\epsilon}-\hat{w}^{\epsilon, h}\right)\right\|_{0, \Omega} \leq C \sqrt{\frac{\epsilon}{h}} \quad \text { and } \quad\left\|\nabla\left(\hat{w}^{\epsilon, h}-w^{\epsilon, h}\right)\right\|_{0, \Omega} \leq C\left(\frac{h^{\prime}}{\epsilon}\right)^{k^{\prime}} .
$$

If oversampling techniques are used and the oversampled domain $S$ satisfies Assumption 3.1 , we have the following result $[11,14]$ :

$$
\left\|\nabla\left(\tilde{w}^{\epsilon}-\hat{w}^{\epsilon, h}\right)\right\|_{h, \Omega} \leq C_{1} \sqrt{\epsilon}+C_{2} \epsilon,
$$

where we recall that $h^{\prime}$ is the local cell size and $k^{\prime}$ is the local order of approximation.

Remark 3.2. By comparing these results with those in $[11,14]$, an extra $h$ term is found in those papers. This term originates in the error between two homogenized solutions. In our case, there is no such error in the homogenized solution since it is exactly $x_{i}$, causing this term to vanish. 
3.2. MsFEM. Denote the $P_{k}$ conforming finite element space associated with the triangulation $\mathcal{T}$ by

$$
V_{h}=\left\{v \in H_{0}^{1}(\Omega):\left.v\right|_{K} \in P_{k}: \forall K \in \mathcal{T}_{h}\right\} .
$$

For a function $v \in H^{1}(\Omega)$, introduce a map $J^{\epsilon, h}$ from $H^{1}(\Omega)$ to $L^{2}(\Omega)$ such that

$$
\left.J^{\epsilon, h} v\right|_{K}=\left.\left(v+\left(w^{\epsilon, h}-x\right) \cdot \nabla v\right)\right|_{K} \text { on each } K \in \mathcal{T},
$$

where $w^{\epsilon, h}$ is obtained by accurately solving the oversampled local problem (3.6) with large enough $S$. define

Let $\left(\Phi_{\ell}^{h}\right)_{\ell=1, \ldots, N_{h}}$ denote a finite element basis of $V_{h}$, where $N_{h}=\operatorname{dim} V_{h}$, and

$$
\Phi_{\ell}^{\epsilon, h}=J^{\epsilon, h} \Phi_{\ell}^{h}, \quad \ell=1, \ldots, N_{h} .
$$

Then the new multiscale finite element space is defined as

$$
V_{h}^{\epsilon}=\operatorname{span}\left\{\Phi_{\ell}^{\epsilon, h}\right\}_{\ell=1, \ldots, N_{h}} .
$$

Note that $V_{h}^{\epsilon} \not \subset H_{0}^{1}(\Omega)$.

Now we can define a Petrov-Galerkin MsFEM: Find $u_{h}^{\epsilon} \in V_{h}^{\epsilon}$ such that

$$
a_{h}^{\epsilon}\left(u_{h}^{\epsilon}, v_{h}\right)=\left(f, v_{h}\right) \quad \forall v_{h} \in V_{h},
$$

where

$$
a_{h}^{\epsilon}(u, v)=\sum_{K \in \mathcal{T}}\left(A^{\epsilon} \nabla u, \nabla v\right)_{K} \quad \forall u \in V_{h}^{\epsilon}, v \in V_{h} .
$$

Or, equivalently, find $u_{h} \in V_{h}$ such that

$$
a_{h}\left(u_{h}, v_{h}\right)=\left(f, v_{h}\right) \quad \forall v_{h} \in V_{h},
$$

where

$$
a_{h}(u, v)=\sum_{K \in \mathcal{T}}\left(A^{\epsilon}\left(\nabla J^{\epsilon, h} u\right), \nabla v\right)_{K} \quad \forall u, v \in V_{h} .
$$

The function $u_{h}$ is an approximation to the homogenized solution $u^{*}$, and $u_{h}^{\epsilon}=$ $J^{\epsilon, h} u_{h} \in V_{h}^{\epsilon}$ is the multiscale finite element approximation to $u^{\epsilon}$.

On an element $K \in \mathcal{T}_{h}$, we have

$$
\nabla\left(J^{\epsilon, h} u_{h}\right)=\left[\nabla w^{\epsilon, h}\right] \nabla u_{h}+\left[\nabla \nabla u_{h}\right]\left(w^{\epsilon, h}-x\right) .
$$

Now define

$$
b_{h}\left(u_{h}, v_{h}\right)=\sum_{K \in \mathcal{T}_{h}}\left(A_{w}^{\epsilon, h} \nabla u_{h}, \nabla v_{h}\right)_{K} \quad \forall u_{h}, v_{h} \in V_{h},
$$

with

$$
\left(A_{w}^{\epsilon, h}\right)_{i j}=\sum_{k=1}^{n} A_{i k}^{\epsilon} \frac{\partial w_{j}^{\epsilon, h}}{\partial x_{k}} \quad \text { or } \quad A_{w}^{\epsilon, h}=A^{\epsilon}\left[\nabla w^{\epsilon, h}\right]
$$


and

$$
c_{h}\left(u_{h}, v_{h}\right)=\sum_{K \in \mathcal{T}_{h}}\left(A^{\epsilon}\left[\nabla\left(\nabla u_{h}\right)\right]\left(w^{\epsilon, h}-x\right), \nabla v_{h}\right)_{K} \quad \forall u_{h}, v_{h} \in V_{h} .
$$

We then have

$$
a_{h}\left(u_{h}, v_{h}\right)=b_{h}\left(u_{h}, v_{h}\right)+c_{h}\left(u_{h}, v_{h}\right) \quad \forall u_{h}, v_{h} \in V_{h} .
$$

When $k=1, c_{h}\left(u_{h}, v_{h}\right)=0$.

Since some functions to be analyzed are not in $H_{0}^{1}(\Omega)$, but only $H^{1}(K)$ for $K \in \mathcal{T}_{h}$, let us define an equivalent broken $H^{1}$-norm as

$$
\|v\|_{h, \Omega}=\left(\sum_{K \in \mathcal{T}_{h}}\|\nabla v\|_{0, K}^{2}\right)^{1 / 2} .
$$

4. Convergence for the periodic case. Let us analyze the multiscale method (3.11), or, equivalently, (3.12) for the periodic case, e.g.,

$$
A^{\epsilon}(x)=A\left(\frac{x}{\epsilon}\right),
$$

where $y \mapsto A(y)$ is a $Y$-periodic function with $Y=(0,1)^{n}$.

We assume that the following inequality holds:

$$
0<h^{\prime}<\epsilon<h<1
$$

for the coarse mesh size $h$, the period $\epsilon$, and the local mesh size $h^{\prime}$.

4.1. Existence and uniqueness of multiscale finite element formulations. In the following we will establish some basic properties of the multiscale finite element formulation, beginning with the following lemma.

Lemma 4.1. When Assumption 3.1 is true, we have

$$
\left\|\nabla w^{\epsilon, h}\right\|_{L^{\infty}(K)} \leq C \quad \forall K \in \mathcal{T}_{h} .
$$

Proof. By the triangle inequality,

$$
\left\|\nabla w^{\epsilon, h}\right\|_{L^{\infty}(K)} \leq\left\|\nabla\left(w^{\epsilon, h}-\hat{w}^{\epsilon, h}\right)\right\|_{L^{\infty}(K)}+\left\|\nabla\left(\tilde{w}^{\epsilon}-\hat{w}^{\epsilon, h}\right)\right\|_{L^{\infty}(K)}+\left\|\nabla \tilde{w}^{\epsilon}\right\|_{L^{\infty}(K)} .
$$

We can bound the three terms as

$$
\begin{aligned}
\left\|\nabla\left(w^{\epsilon, h}-\hat{w}^{\epsilon, h}\right)\right\|_{L^{\infty}(K)} & =\epsilon\left\|\nabla \theta^{\epsilon, K}\right\|_{L^{\infty}(K)}, \\
\mid \nabla\left(\tilde{w}^{\epsilon}-\hat{w}^{\epsilon, h}\right) \|_{L^{\infty}(K)} & \leq C\left(h^{\prime} / \epsilon\right)^{k^{\prime}}, \\
\left\|\nabla \tilde{w}^{\epsilon}\right\|_{L^{\infty}(K)}=\epsilon\left\|\nabla \chi_{i}\right\|_{L^{\infty}(K)} & \leq C .
\end{aligned}
$$

By Assumption 3.1, the result follows.

Lemma 4.2. When Assumption 3.1 is true, for $u_{h}^{\epsilon}=J^{\epsilon, h} u_{h}$, we have

$$
\left\|u_{h}^{\epsilon}\right\|_{h, \Omega} \leq C\left\|\nabla u_{h}\right\|_{0, \Omega} .
$$

Proof. It follows from Lemma 4.1 that

$$
\begin{aligned}
\left\|u_{h}^{\epsilon}\right\|_{h, \Omega} & \leq C\left(\sum_{K \in \mathcal{T}_{h}}\left\|\left[\nabla w^{\epsilon, h}\right] \nabla u_{h}\right\|_{0, K}^{2}\right)^{1 / 2} \leq C\left(\sum_{K \in \mathcal{T}_{h}}\left\|\nabla w^{\epsilon, h}\right\|_{L^{\infty}(K)}^{2}\left\|\nabla u_{h}\right\|_{0, K}^{2}\right)^{1 / 2} \\
& \leq C\left\|\nabla u_{h}\right\|_{0, \Omega},
\end{aligned}
$$


completing the result.

By Lemma 4.2, we obtain the following result.

Lemma 4.3. When Assumption 3.1 is true, we have

$$
a_{h}^{\epsilon}\left(u_{h}^{\epsilon}, v_{h}\right) \leq C\left\|u_{h}^{\epsilon}\right\|_{h, \Omega}\left\|\nabla v_{h}\right\|_{0, \Omega} \quad \forall u_{h}^{\epsilon} \in V_{h}^{\epsilon}, v_{h} \in V_{h}
$$

and

$$
a_{h}\left(u_{h}, v_{h}\right) \leq C\left\|\nabla u_{h}\right\|_{0, \Omega}\left\|\nabla v_{h}\right\|_{0, \Omega} \quad \forall u_{h}, v_{h} \in V_{h} .
$$

Define $\tilde{A}_{w}^{\epsilon}$ as

$$
\left(\tilde{A}_{w}^{\epsilon}\right)_{i j}=\sum_{k=1}^{n} A_{i k}^{\epsilon} \frac{\partial \tilde{w}_{j}^{\epsilon}}{\partial x_{k}}=\sum_{k=1}^{n} A_{i k}^{\epsilon}\left(\delta_{k j}+\frac{\partial \chi_{j}(\mathbf{y})}{\partial y_{k}}\right) \quad \text { or } \quad \tilde{A}_{w}^{\epsilon}=A^{\epsilon}\left[\nabla \tilde{w}^{\epsilon}\right] .
$$

Matrix $\tilde{A}_{w}^{\epsilon}$ is divergence-free, and its average in $Y$ is the constant homogenized matrix $A^{*}[14]$ :

$$
\left\langle\tilde{A}_{w}^{\epsilon}\right\rangle_{Y}=A^{*} \quad \text { and } \quad \nabla \cdot \tilde{A}_{w}^{\epsilon}=0 .
$$

Theorem 4.4. Suppose $\left(h^{\prime} / \epsilon\right)^{k^{\prime}}$ is small enough and (4.1) holds. When Assumption 3.1 is true, there exists a constant $C>0$, independent of $\epsilon$ and $h$, such that

$$
b_{h}\left(v_{h}, v_{h}\right) \geq C\left\|v_{h}\right\|_{1, \Omega}^{2} \quad \forall v_{h} \in V_{h}
$$

and

$$
\sup _{v \in V_{h}} \frac{b_{h}\left(u_{h}, v\right)}{\|v\|_{1, \Omega}} \geq C\left\|u_{h}^{\epsilon}\right\|_{h, \Omega} \quad \forall u_{h}^{\epsilon} \in V_{h}^{\epsilon}
$$

where $u_{h}^{\epsilon}=J^{\epsilon, h} u_{h}$.

Proof. By a simple decomposition, we have

$$
\begin{aligned}
b_{h}\left(v_{h}, v_{h}\right) & =\sum_{K \in \mathcal{T}_{h}}\left(A_{w}^{\epsilon, h} \nabla v_{h}, \nabla v_{h}\right)_{K} \\
& =\sum_{K \in \mathcal{T}_{h}}\left(\tilde{A}_{w}^{\epsilon} \nabla v_{h}, \nabla v_{h}\right)_{K}+\sum_{K \in \mathcal{T}_{h}}\left(\left(A_{w}^{\epsilon, h}-\tilde{A}_{w}^{\epsilon}\right) \nabla v_{h}, \nabla v_{h}\right)_{K} .
\end{aligned}
$$

First, we get a bound for the term $\sum_{K \in \mathcal{T}_{h}}\left(\tilde{A}_{w}^{\epsilon} \nabla v_{h}, \nabla v_{h}\right)_{K}$ using an argument similar to Lemma 3.2 of [14].

By standard homogenization theory (see, e.g., $[9,16]$ ), there exist positive constants $C_{1}$ and $C_{2}$ such that

$$
C_{1}|\xi|^{2} \leq \xi^{t} A^{*} \xi \leq C_{2}|\xi|^{2} \quad \text { for any vector } \xi \in \mathbb{R}^{n} .
$$

Furthermore,

$$
\left(\tilde{A}_{w}^{\epsilon} \nabla v_{h}, \nabla v_{h}\right)_{K}=\left(A^{*} \nabla v_{h}, \nabla v_{h}\right)_{K}+\left(\left(\tilde{A}_{w}^{\epsilon}-A^{*}\right) \nabla v_{h}, \nabla v_{h}\right)_{K} .
$$

Divide $K$ into

$$
K=\left(\bigcup_{Y_{k} \subset K} Y_{k}\right) \bigcup K^{\prime},
$$


where $Y_{k}$ is a periodic cell of $A\left(\frac{x}{\epsilon}\right)$, and $K^{\prime}$ is the difference between $K$ and the union of all $Y_{k}$ in $K$. Since $\left\langle\tilde{A}_{w}^{\epsilon}-A^{*}\right\rangle_{Y_{k}}=0$, for the second term on the right-hand side of $(4.11)$

$$
\left(\left(\tilde{A}_{w}^{\epsilon}-A^{*}\right) \nabla v_{h}, \nabla v_{h}\right)_{K}=\left(\left(\tilde{A}_{w}^{\epsilon}-A^{*}\right) \nabla v_{h}, \nabla v_{h}\right)_{K^{\prime}}+\sum_{Y_{k} \subset K}\left(\left(\tilde{A}_{w}^{\epsilon}-A^{*}\right) \nabla v_{h}, \nabla v_{h}\right)_{Y_{k}} .
$$

For the first term of the right-hand side of (4.12), we have

$\left(\left(\tilde{A}_{w}^{\epsilon}-A^{*}\right) \nabla v_{h}, \nabla v_{h}\right)_{K^{\prime}} \leq \max \left|\left(\tilde{A}_{w}^{\epsilon}-A^{*}\right)\right|\left\|\nabla v_{h}\right\|_{0, K^{\prime}}^{2} \leq C \frac{\epsilon^{2}}{h^{2}} \max \left|\left(\tilde{A}_{w}^{\epsilon}-A^{*}\right)\right|\left\|\nabla v_{h}\right\|_{0, K}^{2}$.

In deriving the last inequality, we have used the fact that $\nabla v_{h}$ is a polynomial vector of fixed degree in $K \in \mathcal{T}_{h}$, and the ratio of the area of $K^{\prime}$ and $K$ is less than $\frac{\epsilon^{2}}{h^{2}}$.

For the second term of the right-hand side of (4.12), recalling that the radius of $Y_{k}$ is $\epsilon$, we recover

$$
\begin{aligned}
\left(\left(\tilde{A}_{w}^{\epsilon}-A^{*}\right) \nabla v_{h}, \nabla v_{h}\right)_{Y_{k}} & \leq \int_{Y_{k}}\left(\nabla v_{h}-c\right)\left(\tilde{A}_{w}^{\epsilon}-A^{*}\right)\left(\nabla v_{h}-c\right) d x \\
& \leq C \epsilon^{2} \max \left|\left(\tilde{A}_{w}^{\epsilon}-A^{*}\right)\right|\left\|\Delta v_{h}\right\|_{0, Y_{k}}^{2} .
\end{aligned}
$$

So, by inverse estimates,

$$
\begin{aligned}
\sum_{Y_{k} \subset K}\left(\left(\tilde{A}_{w}^{\epsilon}-A^{*}\right) \nabla v_{h}, \nabla v_{h}\right)_{Y_{k}} & \leq C \epsilon^{2} \max \left|\left(\tilde{A}_{w}^{\epsilon}-A^{*}\right)\right|\left\|\Delta v_{h}\right\|_{0, K}^{2} \\
& \leq C(\epsilon / h)^{2} \max \left|\left(\tilde{A}_{w}^{\epsilon}-A^{*}\right)\right|\left\|\nabla v_{h}\right\|_{0, K}^{2}
\end{aligned}
$$

Summing up, using that by (4.1), $\frac{\epsilon}{h}<1$, we have

$$
C_{1}\left\|\nabla v_{h}\right\|_{0, K}^{2} \leq \sum_{K \in \mathcal{T}_{h}}\left(\tilde{A}_{w}^{\epsilon} \nabla v_{h}, \nabla v_{h}\right)_{K} \leq C_{2}\left\|\nabla v_{h}\right\|_{0, K}^{2} \quad \forall v_{h} \in V_{h}, K \in \mathcal{T}_{h} .
$$

Let us now consider the second term on the right-hand side of (4.9). On an element $K$,

$(4.15)$

$$
\tilde{A}_{w}^{\epsilon}-A_{w}^{\epsilon, h}=A^{\epsilon}\left(\left[\nabla \tilde{w}^{\epsilon}\right]-\left[\nabla \tilde{w}^{\epsilon, h}\right]\right)=A^{\epsilon}\left(\left[\nabla \tilde{w}^{\epsilon}\right]-\left[\nabla \hat{w}^{\epsilon, h}\right]\right)+A^{\epsilon}\left(\left[\nabla \hat{w}^{\epsilon, h}\right]-\left[\nabla \tilde{w}^{\epsilon, h}\right]\right) .
$$

The two terms on the right-hand side of (4.15) can be bounded by

$$
\left\|\mathcal{A}^{\epsilon}\left(\left[\nabla \tilde{w}^{\epsilon}\right]-\left[\nabla \hat{w}^{\epsilon, h}\right]\right)\right\|_{L^{\infty}(K)} \leq \epsilon\left\|A^{\epsilon} \nabla \theta^{\epsilon, K}\right\|_{L^{\infty}(K)} \leq \epsilon C\left\|\nabla \theta^{\epsilon, K}\right\|_{L^{\infty}(K)}
$$

and

$$
\left\|A^{\epsilon}\left(\left[\nabla \hat{w}^{\epsilon, h}\right]-\left[\nabla \tilde{w}^{\epsilon, h}\right]\right)\right\|_{L^{\infty}(K)} \leq C\left(h^{\prime}\right)^{k^{\prime}}\left|\hat{w}^{\epsilon, h}\right|_{W^{\infty, k^{\prime}+1}(K)} \leq C\left(h^{\prime} / \epsilon\right)^{k^{\prime}} .
$$

Hence

$$
\left\|\tilde{A}_{w}^{\epsilon}-A_{w}^{\epsilon, h}\right\|_{L^{\infty}(K)} \leq C\left(\epsilon+\left(h^{\prime} / \epsilon\right)^{k^{\prime}}\right) .
$$

Therefore, if Assumption 3.1 is true and $\epsilon / h$ is bounded, the second term on the right-hand side of (4.9) can be bounded by the first term of the right-hand side of (4.9), provided $\left(h^{\prime} / \epsilon\right)^{k^{\prime}}$ is small enough. This proves (4.7). 
By Lemma 4.2, with $v_{h}=u_{h}$,

$$
b_{h}\left(u_{h}, u_{h}\right) \geq C\left\|\nabla u_{h}\right\|_{1, \Omega}^{2} \geq C\left\|\nabla u_{h}\right\|_{1, \Omega}\left\|u_{h}^{\epsilon}\right\|_{h, \Omega},
$$

completing the proof of (4.8).

Remark 4.5. For the nonoversampling case $S=K$, we may still assume that the coercivity results for Lemma 4.2 is true for small enough $\epsilon$. Naturally, the constant will be smaller than in the oversampling case, but we may still assume it is bounded away from zero. The inf-sup condition is much worse in the nonoversampling case and may blow up since the bound $\left\|u_{h}^{\epsilon}\right\|_{h, \Omega} \leq C\left\|u_{h}\right\|_{1, \Omega}$ may not be valid.

TheOREM 4.6. With the same assumption as in Theorem 4.4, there exists a constant $C>0$ such that

$$
a_{h}\left(u_{h}, u_{h}\right) \geq C\left\|u_{h}\right\|_{1, \Omega}^{2} \quad \forall u_{h} \in V_{h}
$$

and

$$
\sup _{v \in V_{h}} \frac{a_{h}^{\epsilon}\left(u_{h}^{\epsilon}, v\right)}{\|v\|_{1, \Omega}} \geq C\left\|u_{h}^{\epsilon}\right\|_{h, \Omega} \quad \forall u_{h}^{\epsilon} \in V_{h}^{\epsilon} .
$$

Proof. For $k=1, a_{h}$ and $b_{h}$ are equivalent, so we consider only the case of $k \geq 2$. Note that

$$
\begin{aligned}
\left\|w^{\epsilon, h}-x\right\|_{L^{\infty}(K)} & \leq\left\|w^{\epsilon, h}-x\right\|_{L^{\infty}(K)}+\left\|w^{\epsilon, h}-\tilde{w}^{\epsilon}\right\|_{L^{\infty}(K)}+\left\|\tilde{w}^{\epsilon}-x\right\|_{L^{\infty}(K)} \\
& \leq \epsilon\left\|\theta^{\epsilon, K}\right\|_{L^{\infty}(K)}+C\left(\frac{h^{\prime}}{\epsilon}\right)^{k^{\prime}+1}+C \epsilon \leq C\left(\epsilon+\left(\frac{h^{\prime}}{\epsilon}\right)^{k^{\prime}+1}\right) .
\end{aligned}
$$

By the above bound and an inverse estimate, we recover

$$
\begin{aligned}
c_{h}\left(u_{h}, v_{h}\right) & =\sum_{K \in \mathcal{T}_{h}}\left(A^{\epsilon}\left[\nabla\left(\nabla u_{h}\right)\right]\left(w^{\epsilon, h}-x\right), \nabla v_{h}\right)_{K} \\
& \leq C \sum_{K \in \mathcal{T}}\left\|w^{\epsilon, h}-x\right\|_{L^{\infty}(K)} \frac{1}{h}\left\|\nabla u_{h}\right\|_{0, K}\left\|\nabla v_{h}\right\|_{0, K} \\
& \leq C \frac{\epsilon+\left(\frac{h^{\prime}}{\epsilon}\right)^{k^{\prime}+1}}{h}\left\|\nabla u_{h}\right\|_{0, K}\left\|\nabla v_{h}\right\|_{0, K} .
\end{aligned}
$$

The result follows from Theorem 4.4.

Remark 4.7. It is tempting to use the formulation based on the bilinear form $b$ only. Suppose $u_{h, a}$ is the solution of (3.12) and $u_{h, b} \in V_{h}$ is the solution of

$$
b_{h}\left(u_{h, b}, v_{h}\right)=\left(f, v_{h}\right) \quad \forall v_{h} \in V_{h} .
$$

Let $z_{h}=u_{h, a}-u_{h, b}$; then

$$
C\left\|z_{h}\right\|_{1, \Omega}^{2} \leq b_{h}\left(z_{h}, z_{h}\right)=-c_{h}\left(u_{h, a}, z_{h}\right) \leq C \frac{\epsilon+\left(\frac{h^{\prime}}{\epsilon}\right)^{k^{\prime}+1}}{h}\left\|\nabla u_{h, a}\right\|_{1, \Omega}\left\|z_{h}\right\|_{1, \Omega} .
$$

For $k \geq 2$, the difference between the two solutions may be as bad as of order $\frac{\epsilon+\left(\frac{h^{\prime}}{\epsilon}\right)^{k^{\prime}+1}}{h}$. Hence, the method based on the $b$ form alone is not recommended. 
4.2. Convergence proof of MsFEMs. Let us first recall a slightly altered standard result.

Lemma 4.8 (generalized Cea's lemma). Let $u^{\epsilon}$ be the solution of (2.1) and $u_{h}^{\epsilon}$ the solution of (3.11). Taking the assumptions of Theorem 4.4 to be true, there exists a constant $C$ such that

$$
\left\|u^{\epsilon}-u_{h}^{\epsilon}\right\|_{h, \Omega} \leq C \inf _{v_{h}^{\epsilon} \in V_{h}^{\epsilon}}\left\|u^{\epsilon}-v_{h}^{\epsilon}\right\|_{h, \Omega} .
$$

Proof. Note that the following Galerkin orthogonality holds:

$$
a_{h}^{\epsilon}\left(u_{h}^{\epsilon}-u^{\epsilon}, v_{h}\right)=0 \quad \forall v_{h} \in V_{h} .
$$

Then, by a standard argument (see, e.g., Lemma 3.7 of [6]), we have the desired result.

Remark 4.9. The constant $C$ in the above lemma depends on the inf-sup constant, so we must ensure that the size of the oversampling domain $S$ is big enough.

For a sufficiently smooth function $v$ with meaningful nodal values, define $\Pi_{h}$ to be the standard $V_{h}$-interpolation operator,

$$
\Pi_{h} v(x)=\sum_{\ell=1}^{N_{h}} v\left(n_{\ell}\right) \Phi_{\ell}^{h}(x) \in V_{h},
$$

where $n_{\ell}$ is the node associated with the $P_{k}$ finite elements. The operator $\Pi_{h}^{\epsilon}$ is defined as

$$
\Pi_{h}^{\epsilon} v=J^{\epsilon, h}\left(\Pi_{h} v\right)=\sum_{\ell=1}^{N_{h}} v\left(n_{\ell}\right) \Phi_{\ell}^{\epsilon, h}(x) \in V_{h}^{\epsilon} .
$$

TheOrem 4.10. Let $u^{\epsilon}$ be the solution of (2.1). Assume $u_{h}^{\epsilon}$ is the solution of the Petrov-Galerkin formulation (3.11) with the oversampling domain $S$ satisfying Assumption 3.1. Then the following error estimate holds:

$$
\left\|u^{\epsilon}-u_{h}^{\epsilon}\right\|_{h, \Omega} \leq C\left(h^{k}\|f\|_{0}+\sqrt{\epsilon}+\left(\frac{h^{\prime}}{\epsilon}\right)^{k^{\prime}}\right) .
$$

Proof. Let $u^{*}$ be the exact solution of the homogenized problem (2.5), and choose $v_{h}^{\epsilon}=\Pi_{h}^{\epsilon} u^{*}$. Then we have

$$
\left\|u^{\epsilon}-u_{h}^{\epsilon}\right\|_{h, \Omega} \leq C\left\|u^{\epsilon}-\Pi_{h}^{\epsilon} u^{*}\right\|_{h, \Omega} .
$$

Define two operators $J^{\epsilon}$ and $\tilde{J}^{\epsilon}$ as

$\left.J^{\epsilon} v\right|_{K}=\left.\left(v+\left(\tilde{w}^{\epsilon}-x\right) \cdot \nabla v\right)\right|_{K},\left.\quad \widehat{J}^{\epsilon} v\right|_{K}=\left.\left(v+\left(\hat{w}^{\epsilon, h}-x\right) \cdot \nabla v\right)\right|_{K}$ on each $K \in \mathcal{T}_{h}$.

We bound (4.23) as

$$
\begin{aligned}
\left\|u^{\epsilon}-\Pi_{h}^{\epsilon} u^{*}\right\|_{h, \Omega} \leq & \left\|u^{\epsilon}-J^{\epsilon} u^{*}\right\|_{h, \Omega}+\left\|J^{\epsilon}\left(u^{*}-\Pi_{h} u^{*}\right)\right\|_{h, \Omega} \\
& +\left\|\left(J^{\epsilon}-\widehat{J}^{\epsilon, h}\right) \Pi_{h} u^{*}\right\|_{h, \Omega}+\left\|\left(\widehat{J}^{\epsilon, h}-J^{\epsilon, h}\right) \Pi_{h} u^{*}\right\|_{h, \Omega .}
\end{aligned}
$$

The first term on the right-hand side of (4.25) is bounded by $C \sqrt{\epsilon}$ using Lemma 2.13 of [3]. 
By the definition of the broken $H^{1}$-norm, the second term of the right-hand side of (4.25) is bounded as

$$
\left\|J^{\epsilon}\left(u^{*}-\Pi_{h} u^{*}\right)\right\|_{h, \Omega}=\left(\sum_{K \in \mathcal{T}_{h}}\left\|\nabla\left(\left.J^{\epsilon}\right|_{K}\left(u^{*}-\Pi_{h} u^{*}\right)\right)\right\|_{0, K}^{2}\right)^{1 / 2} .
$$

On each element $K \in \mathcal{T}_{h}$, by standard interpolation results of $P_{k}$ finite elements,

$$
\begin{aligned}
\left\|\nabla\left(J^{\epsilon}\left(u^{*}-\Pi_{h} u^{*}\right)\right)\right\|_{0, K} & \left.\leq\left\|\nabla J^{\epsilon}\right\|_{L^{\infty}(K)} \| \nabla\left(u^{*}-\Pi_{h} u^{*}\right)\right) \|_{0, K} \\
& \left.\leq C h^{k}\left\|\nabla J^{\epsilon}\right\|_{L^{\infty}(K)} \| \nabla\left(u^{*}-\Pi_{h} u^{*}\right)\right) \|_{k+1, K} .
\end{aligned}
$$

The third term of in the right-hand side of (4.25) is bounded by

$$
\left\|\left(J^{\epsilon}-\widehat{J}^{\epsilon, h}\right) \Pi_{h} u^{*}\right\|_{h, \Omega} \leq\left\|\nabla \Pi_{h} u^{*}\right\|_{L^{\infty}(\Omega)}\left\|, \nabla_{h}\left(\tilde{w}^{\epsilon}-\hat{w}^{\epsilon, h}\right)\right\|_{0, \Omega},
$$

and the fourth term of in the right-hand side of (4.25) is bounded by

$$
\left\|\left(\widehat{J}^{\epsilon, h}-J^{\epsilon, h}\right) \Pi_{h} u^{*}\right\|_{h, \Omega} \leq\left\|\nabla \Pi_{h} u^{*}\right\|_{L^{\infty}(\Omega)}\left\|\nabla_{h}\left(w^{\epsilon, h}-\hat{w}^{\epsilon, h}\right)\right\|_{0, \Omega} .
$$

By (3.9), this proves the theorem.

4.3. Estimates on the homogenized solutions. In the following we prove an estimate for the homogenized solution in the $H^{1}$-norm similar to Theorem 3.4 of [14].

TheOREM 4.11. Assume that $u^{*}$ belongs to $H^{3}(\Omega) \cap W^{2, \infty}(\Omega)$ and $\partial \Omega$ is $C^{0,1}$. Under Assumption 3.1, we have the following estimate for the solution $u_{h}$ of (3.12):

$$
\left\|u_{h}-u^{*}\right\|_{1, \Omega} \leq C_{1} \epsilon+C_{2} h^{k}+C_{3} \epsilon|\ln h|^{1 / 2}+C_{4}\left(\frac{h^{\prime}}{\epsilon}\right)^{k^{\prime}} .
$$

Proof. The triangle inequality yields

$$
\left\|u_{h}-u^{*}\right\|_{1, \Omega} \leq\left\|u_{h}-\Pi_{h} u^{*}\right\|_{1, \Omega}+\left\|\Pi_{h} u^{*}-u^{*}\right\|_{1, \Omega} .
$$

Denote $u_{h}-\Pi_{h} u^{*}$ by $v$; then

$$
C\left\|u_{h}-\Pi_{h} u^{*}\right\|_{1}^{2} \leq a_{h}\left(u_{h}-\Pi_{h} u^{*}, v\right)=a_{h}\left(u_{h}-u^{*}, v\right)+a_{h}\left(u^{*}-\Pi_{h} u^{*}, v\right) .
$$

The term $a_{h}\left(u^{*}-\Pi_{h} u^{*}, v\right)$ is handled by continuity of $a_{h}$ and the approximation property of $\Pi_{h}$.

Since

$$
a_{h}\left(u_{h}, v_{h}\right)=\left(f, v_{h}\right)=\left(A^{*} \nabla u^{*}, \nabla v\right) \quad \forall v_{h} \in V_{h},
$$

for the term $a_{h}\left(u_{h}-u^{*}, v\right)$, we have

$$
a_{h}\left(u_{h}-u^{*}, v\right)=a_{h}\left(u_{h}, v\right)-a_{h}\left(u^{*}, v\right)=\left(A^{*} \nabla u^{*}, \nabla v\right)-a_{h}\left(u^{*}, v\right)=I_{1}+I_{2},
$$

where

$$
I_{1}=\left(A^{*} \nabla u^{*}, \nabla v\right)-\left(\tilde{A}_{w}^{\epsilon} \nabla u^{*}, \nabla v\right)
$$

and

$$
I_{2}=\left(\tilde{A}_{w}^{\epsilon} \nabla u^{*}, \nabla v\right)-\sum_{K \in \mathcal{T}_{h}}\left(A^{\epsilon} \nabla\left(J^{\epsilon, h} u^{*}\right), \nabla v\right)_{K}
$$


From the analysis of Theorem 3.4 in [14], we recall that

$$
I_{1} \leq\left(C_{1} \epsilon+C_{2} h^{k}+C_{3} \epsilon(\ln h)^{1 / 2}\right)\|v\|_{1, \Omega}
$$

The order of $h$ is $k$ instead of 1 as in Theorem 3.4 of [14] since we consider polynomials of degree $k$.

Now, consider the restriction of $I_{2}$ to an element $K$,

$$
\left(\tilde{A}_{w}^{\epsilon} \nabla u^{*}-A^{\epsilon} \nabla\left(J^{\epsilon, h} u^{*}\right), \nabla v\right)_{K}=I_{3}+I_{4}
$$

with

$$
I_{3}=\left(\left(\tilde{A}_{w}^{\epsilon}-A_{w}^{\epsilon, h}\right) \nabla u^{*}, \nabla v\right)_{K} \leq C\left(\epsilon+\left(h^{\prime} / \epsilon\right)^{k^{\prime}}\right)\left\|\nabla u^{*}\right\|_{0, K}\|\nabla v\|_{0, K}
$$

and

$$
I_{4}=-\left(A^{\epsilon}\left[\nabla \nabla u^{*}\right]\left(w^{\epsilon, h}-x\right), \nabla v\right)_{K} \leq C\left(\epsilon+\left(h^{\prime} / \epsilon\right)^{k^{\prime}+1}\right)\left\|\nabla \nabla u^{*}\right\|_{0, K}\|\nabla v\|_{0, K} .
$$

Estimates (4.16) and (4.19) are used in the derivation of the bounds of $I_{3}$ and $I_{4}$. Summing up all the bounds yields the final estimate and completes the proof.

4.4. $L^{2}$ estimates for the multiscale finite element solutions. In the following, we derive two bounds for the $L^{2}$-norm estimates of $u^{\epsilon}$, the solution of (2.1), and $u_{h}^{\epsilon}$, the solution of (3.11). This is done under the assumptions of Theorem 4.4.

Theorem 4.12. Assume $u^{*}$ belongs to $H^{3}(\Omega) \cap W^{2, \infty}(\Omega)$ and $\partial \Omega$ is $C^{0,1}$. Under Assumption 3.1, we have the following estimate for the solution $u_{h}^{\epsilon}$ of (3.11):

$$
\left\|u_{h}^{\epsilon}-u^{\epsilon}\right\|_{0, \Omega} \leq C\left(\epsilon+h^{k}+\epsilon|\ln h|^{1 / 2}+\left(\frac{h^{\prime}}{\epsilon}\right)^{k^{\prime}}\right) .
$$

Proof. The proof we have follows that of Theorem 3.5 of [14]. By Theorem 4.11 and triangle inequalities,

$$
\begin{aligned}
\left\|u_{h}^{\epsilon}-u^{\epsilon}\right\|_{0, \Omega} & \leq\left\|u_{h}-u^{*}\right\|_{0, \Omega}+\left\|u_{h}^{\epsilon}-u^{*}\right\|_{0, \Omega} \\
& \leq\left\|u_{h}-u^{*}\right\|_{0}+\left\|\left(w^{\epsilon, h}-x\right) \nabla u_{h}\right\|_{0}+C \epsilon \\
& \leq\left\|u_{h}-u^{*}\right\|_{1}+C\left(\epsilon+\left(h^{\prime} / \epsilon\right)^{k+1}\right) \\
& \leq C\left(\epsilon+h^{k}+\epsilon|\ln h|^{1 / 2}+\left(\frac{h^{\prime}}{\epsilon}\right)^{k^{\prime}}\right) .
\end{aligned}
$$

As suggested in [13], with the $H^{1}$ error estimate (4.22), we can get another error estimate in the $L^{2}$-norm given by the standard Aubin-Nitsche trick to increase one more order of the right hand side $f$. The proof is skipped.

THEOREM 4.13. Under Assumption 3.1, we have the following estimate for the solution $u_{h}^{\epsilon}$ of $(3.11)$ :

$$
\left\|u_{h}^{\epsilon}-u^{\epsilon}\right\|_{0, \Omega} \leq C\left(h^{k+1}\|f\|_{0}+\sqrt{\epsilon}+\left(\frac{h^{\prime}}{\epsilon}\right)^{k^{\prime}}\right) .
$$

Remark 4.14. The above two $L^{2}$ estimations are both valid, and we can choose the smaller bound. In the extreme case, when the error of the coarse problem $\left(h^{k+1}\right)$ 
is much bigger, we should use the result of Theorem 4.13, and when the multiscale error is dominant ( $\sqrt{\epsilon}$ is big), we should use the result of Theorem 4.12.

Remark 4.15. We have established only the results for the Petrov-Galerkin formulation with oversampling. If a standard Galerkin formulation is used, the MsFEMs with the new basis functions are nonconforming, and the optimal convergence results similar to those in [11] can be shown. If oversampling is not used and standard Galerkin method is applied, we can still recover results similar to those of [3]. However, in both cases, these results are inferior to those obtained above.

5. Numerical tests. We present in the following a number of computational tests to validate the analysis presented above. The main goal is to show that the analysis is sharp and to illustrate the interplay between the different parameters in the high-order MsFEM.

We consider the numerical experiment in [3] with a smooth scalar conductivity tensor: $A^{\epsilon}=a(x / \epsilon) I$, where

$$
a(x / \epsilon)=\frac{1}{(2+P \sin (2 \pi x / \epsilon))(2+P \sin (2 \pi y / \epsilon))},
$$

where $P=1.8$ and $f=1$. All experiments are done on the unit square domain $[0,1] \times[0,1]$, uniformly triangulated with a coarse mesh of size $h$. Each coarse element is furthermore uniformly triangulated with the fine mesh of size $h^{\prime}<\epsilon / 10$ to recover the local solution.

In Figure 1, we plot $w_{1}^{\epsilon, h}(x / \epsilon)-x$ and $w_{2}^{\epsilon, h}(y / \epsilon)-y$ for $\epsilon=0.01$ on one triangular element (solved by the $P_{2}$ element). The oscillatory feature of the oscillatory function $w^{\epsilon, h}$ is clearly observed.
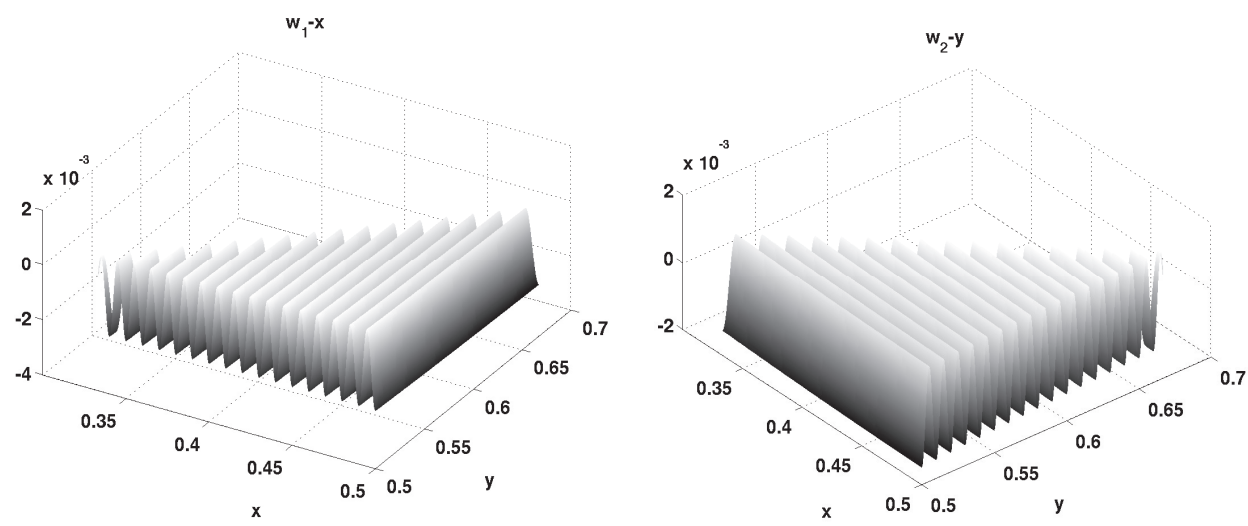

FIG. 1. Illustrations of $w_{1}^{\epsilon, h}(x / \epsilon)-x$ and $w_{2}^{\epsilon, h}(y / \epsilon)-y$ for $\epsilon=0.01$ on one triangular element.

5.1. Convergence of the homogenized solution. We first compare the numerical homogenized solution with the approximate homogenized solution. In this case, the homogenized conductivity is known exactly as $A^{*}=1 /\left(2\left(4-P^{2}\right)^{1 / 2}\right)[3]$.

In Figure 2 we show the convergence of the $L^{2}$ and the $H^{1}$ errors of the homogenized solution $u_{h}$, where $N$ is the number of degrees of freedom in each dimension. 

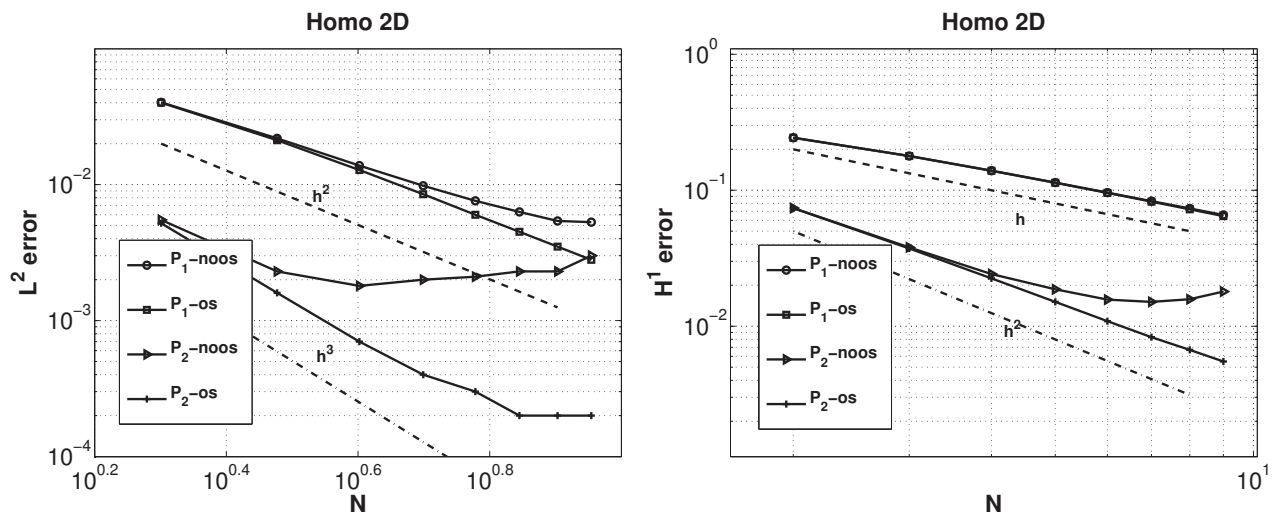

FIG. 2. $L^{2}$ and $H^{1}$ errors of $u^{*}-u_{h}$ by the $P_{1}$ and $P_{2}$ approximations in two dimensions with (os) and without (noos) oversampling approaches for $\epsilon=0.01$.
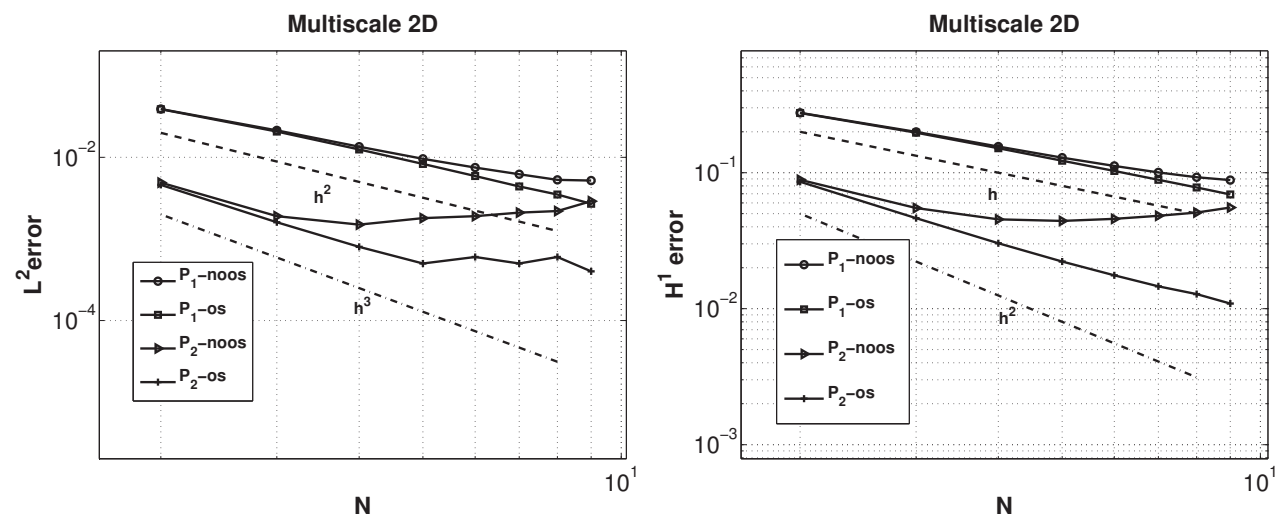

FIG. 3. $L^{2}$ and $H^{1}$ errors of $u-u^{m s}$ by the $P_{1}$ and $P_{2}$ approximations in two dimensions with (os) and without (noos) oversampling approaches for $\epsilon=0.01$.

We see the optimal order of convergence in the $H^{1}$-norm before the error caused by $\epsilon$ begins to dominate. For the $P_{2}$ approximation, we also clearly observe the influence of oversampling on the convergence in Figure 2.

5.2. Convergence of the multiscale solution. To explore the convergence of the multiscale solution, we repeat the numerical experiments above but now compare the reference solution computed by a first-order finite element obtained using a very fine mesh. In Figure 3, we show the convergence of the $L^{2}$ and $H^{1}$ errors of the multiscale solution $u^{m s}$ for $\epsilon=0.01$, where $N$ is the number of degrees of freedom in each dimension, and $h=1 / N>\epsilon$. In the $P_{1}$ case we observe first-order convergence in the $H^{1}$ error.

For the $P_{2}$ case, approximate third-order convergence in the $L^{2}$ norm is observed when the oversampling approach is used. In Figure 4, we also show the influence of $\epsilon$ on the convergence. We also compare the Galerkin and Petrov-Galerkin approaches for the $P_{2}$ case in Figure 5. We observed that before the error plateau caused by $\epsilon$, the Petrov-Galerkin approach behaves better than the standard Galerkin approach. For 

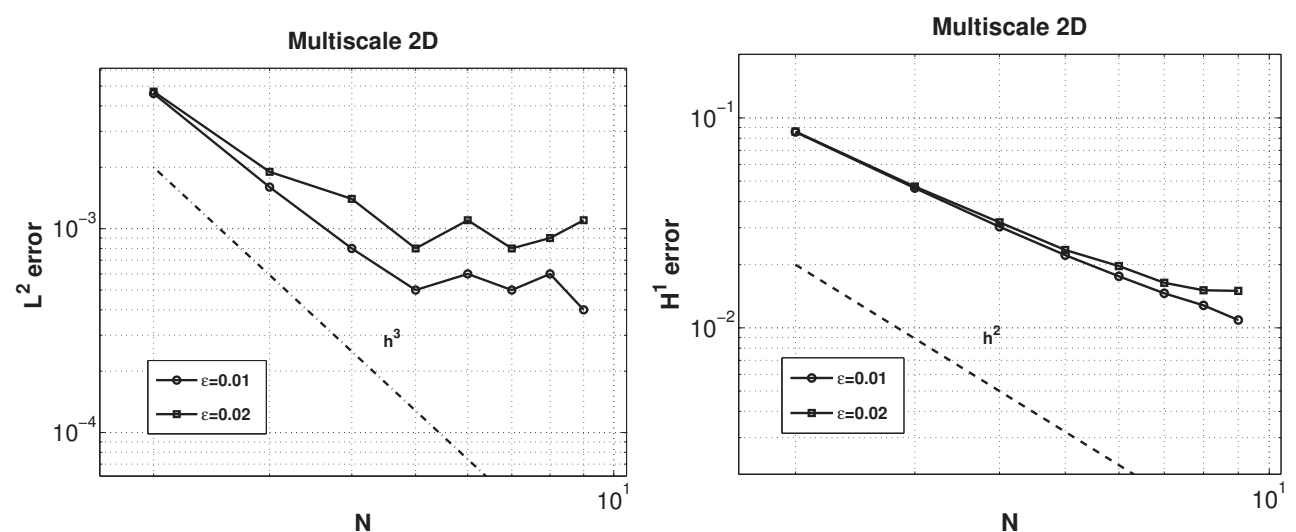

FIG. 4. Error $u-u_{h}^{m s}$ by the $P_{2}$ approximation in two dimensions with oversampling for $\epsilon=0.01,0.02$.
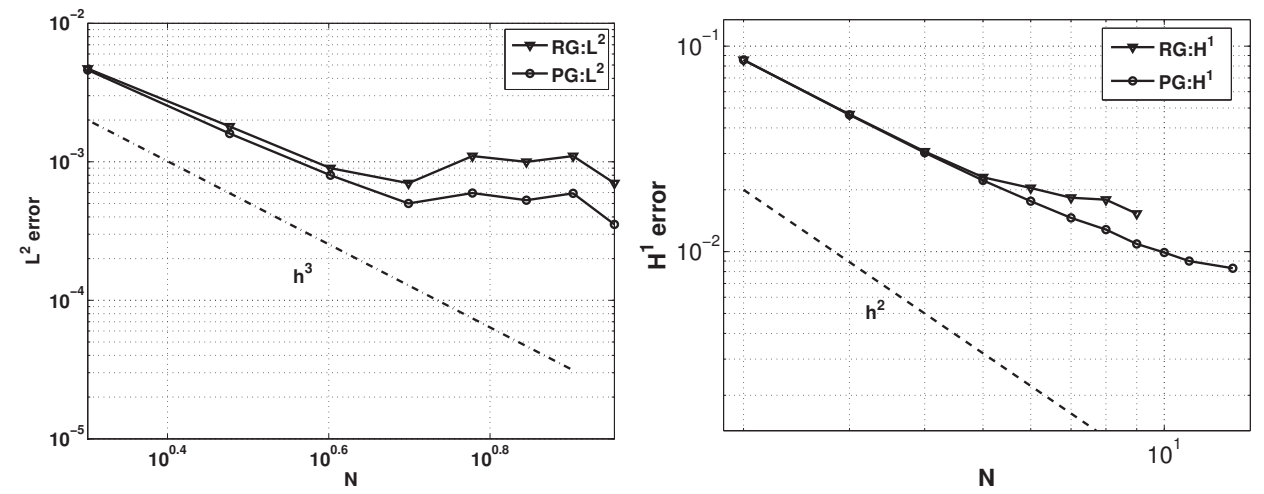

FIG. 5. Comparison of the error $u-u_{h}^{m s}$ using second-order Galerkin (RG) and Petrov-Galerkin $(P G)$ approximations with oversampling approaches in two dimensions for $\epsilon=0.01$.

$\epsilon=0.02$, we took $h^{\prime}=h / 250<\epsilon / 10$; while for $\epsilon=0.01$, we took $h^{\prime}=h / 500<\epsilon / 10$, where $h=1 / N>\epsilon$. For oversampling, we take the size of the oversampling element $h_{o}=4 h$.

6. Concluding remarks. In this paper, we develop a new high-order MsFEM for elliptic problems with highly oscillating coefficients. A more explicit multiscale finite element space is constructed, inspired by Allaire and Brizzi's method [3]. Optimal error estimates are proved in the case of periodically oscillating coefficients.

The method developed in this paper is being applied by the authors to Helmholtz equations with oscillating coefficients, where high-order methods are naturally required.

Due to the nature of similarity of local problems, we are also developing reduced basis MsFEMs for the methods developed in [3] and this paper to reduce the computation cost.

Note that the oversampling technique is very expensive computationally; to reduce the cost, the authors are presently exploring an adaptive methods for the oversampling problem based on local a posteriori error estimates. 


\section{REFERENCES}

[1] A. Abdulle, W. E., B. Engquist, And E. Vanden-Eijnden, The heterogeneous multiscale method, Acta Numer., 21 (2012), pp. 1-87.

[2] G. Allaire, Shape Optimization by the Homogenization Method, Springer-Verlag, New York, 2002.

[3] G. Allaire and R. BrizzI, A multiscale finite element method for numerical homogenization, Multiscale Model. Simul., 4 (2005), pp. 790-812.

[4] T. Arbogast And K. J. Boyd, Subgrid upscaling and mixed multiscale finite elements, SIAM J. Numer. Anal., 44 (2006), pp. 1150-1171.

[5] A. Bensoussan, J.-L. Lions, and G. Papanicolaou, Asymptotic Analysis for Periodic Structures, Stud. Math. Appl. 5, North-Holland, Amsterdam, New York, 1978.

[6] D. Braess, Finite Elements: Theory, Fast Solvers and Applications in Solid Mechanics, 3rd ed., Cambridge University Press, Cambridge, UK, 2007.

[7] Z. Chen, M. Cui, T. Y. Savchuk, And X. YU, The multiscale finite element method with nonconforming elements for elliptic homogenization problems, Multiscale Model. Simul., 7 (2008), pp. 517-538.

[8] Z. Chen And T. Y. Hou, A mixed multiscale finite element method for elliptic problems with oscillating coefficients, Math. Comp., 72 (2003), pp. 541-576.

[9] D. Cioranescu and P. Donato, An Introduction to Homogenization, Oxford Lecture Ser. Math. Appl. 17, The Clarendon Press, Oxford University Press, New York, 1999.

[10] Y. Efendiev And T. Y. Hou, Multiscale Finite Element Methods: Theory and Applications, Springer, New York, 2009.

[11] Y. R. Efendiev, T. Y. Hou, And X.-H. Wu, Convergence of a nonconformal multiscale finite element method, SIAM J. Numer. Anal., 37 (2000), pp. 888-910.

[12] T. Y. Hou And X-H. Wu, A multiscale finite element method for elliptic problems in composite materials and porous media, J. Comput. Phys., 134 (1997), pp. 169-189.

[13] T. Y. Hou, X.-H. Wu, AND Z. CAI, Convergence of a multiscale finite element method for elliptic problems with rapidly oscillating coefficients, Math. Comp., 68 (1999), pp. 913943.

[14] T. Y. Hou, X.-H. Wu, AND Y. Zhang, Removing the cell resonance error in the multiscale finite element method via a Petrov-Galerkin formulation, Commun. Math. Sci., 2 (2004), pp. $185-205$.

[15] T. J. R. Hughes, G. R. Feijoo, L. Mazzei, And J.-B. Quincy, The variational multiscale method - a paradigm for computational mechanics, Comput. Methods Appl. Mech. Engrg., 166 (1998), pp. 3-24.

[16] V. Jikov, S. KozLov, And O. Oleinik, Homogenization of Differential Operators and Integral Functionals, Springer, Berlin, 1995.

[17] A. M. Matache, I. BabušKa, And C. Schwab, Generalized p-FEM in homogenization, Numer. Math., 86 (2000), pp. 319-375.

[18] F. Murat and L. Tartar, H-convergence, in Topics in the Mathematical Modelling of Composite Materials, Progr. Nonlinear Differential Equations Appl. 31, Birkhäuser Boston, Boston, MA, 1997, pp. 21-43.

[19] H. Owhadi and L. Zhang, Metric-based upscaling, Comm. Pure Appl. Math., 60 (2007), pp. $675-723$.

[20] L. TARTaR, The General Theory of Homogenization. A Personalized Introduction, Lect. Notes Unione Mat. Ital. 7, Springer-Verlag, Berlin; UMI, Bologna, 2009. 